\title{
A Survey of Digital Image Watermarking Optimization Algorithms Inspired by Nature
}

\author{
Komal Sinsinwar ${ }^{1}$, SPS Chauhan ${ }^{2}$ \\ ${ }^{1}$ School of Computing Science and Engineering, Galgotias University, Uttar Pradesh, India \\ ${ }^{2}$ Assistant Professor, School of Computing Science and Engineering, Galgotias University, Uttar Pradesh, India
}

\begin{abstract}
Real world problems in the recent years have become more complex, hence more powerful optimization techniques are needed to solve these complex and unsolvable problems. These algorithms provide a protean and conditional solution. In this paper a survey of the algorithms inspired by nature which can be used to generate optimal watermarked image. Firefly Algorithm (FA), Modified Firefly Algorithm (MFA), Cuttlefish Algorithm(CA), Bat Algorithm(BA), Artificial Bee Colony, Seed Based Plant Propagation Algorithm, are considered to generate the optimal digital watermarking.
\end{abstract}

Keywords: Nature based Algorithms, Optimal Watermarking, and Complex Problems

\section{Introduction}

With the ever changing world of technology, need for adaptations in the existing models to deal with the today"s problems in the field of computer Science are needed. Watermarking is one such problem which needs to be performed well so that the authentication, security and copyright of the data which is distributed over the internetare intact. Watermarking is a process of securing the data from illegal actions, by adding an extra signal, called watermark signal to the original data. The data could be an image, video or audio. This watermark signal is embedded to the original data in such a way that it doesn et change the original data in respect of its appearance and overall structure. Also this new watermarked data should be susceptible to any change performed on that data afterwards. The watermark signal should be robust enough to withstand majority of attacks such as compression, Gaussian filtering, scaling, etc.

The Digital image watermarking process should performed be in such a way that the watermark can be extracted later without doing any damage to the original image. This watermark process should deliver robustness, transparency, security of the watermark image. Watermarking can be classified into many subcategories based on their visibility, domain, and durability. In terms of domain, watermarking can be divided into two parts: spatial domain and frequency domain.

The watermark is embedded by directly altering the pixel value of the original image in case of spatial domain technique. This is rather a less complex and easy to implement. But the disadvantage of fragility of the resulted watermarked image to the various attacks, overshadow this advantageous property of spatial domain watermarking scheme. On the other hand, frequency domain technique is more robust in terms of the watermark image and less fragile to the attacks. Frequency domain scheme, also known as transform domain, modulates certain frequencies in a particular domain, such as Discrete Cosine Transform (DCT), Discrete Wavelet Transform (DWT), Singular Value Decomposition (SVD) and Discrete Fourier Transform
(DFT). The watermark is then embedded at these modulated coefficients of the frequencies. Despite the fact that frequency domain techniques provide more robustness and present more information, it is costly to implement and more complex than spatial domain because of extra complex computations it have. Also the watermark strength is greater in case of frequency domain scheme. A watermarking strength can be defined as the amount of information of the watermark is embedded in the host image. Therefore, frequency domain techniques are used more often in watermarking.

The watermarking embedding procedure involves the scaling factors which are used to determine the strength of the watermark. These scaling factors should be chosen in such a way that it delineate the deformities in the watermarked image. To do so, we need multiple scaling factors rather than single scaling factor. The selection of multiple scaling factors (MSF) is very critical as it determines the overall structure, imperceptibility and robustness of the watermarked image. This selection of $\mathrm{MSF}^{\mathrm{ee}} \mathrm{S}$ is an optimization problem which can be solved by many optimization algorithms such as Firefly Algorithm, Cuttlefish Algorithm, Bat Algorithm, Artificial Bee Colony algorithm etc. The Nature based Algorithms have emerged as a strong solution to these optimization problems in the field of digital image watermarking. This paper presents the survey of the latest Nature based Algorithms which are proved to solve many complex optimization problems of digital image watermarking. The rest of the paper is constituted of the following sections: Section II gives the brief overview of the algorithms. The conclusion is drawn in section IV.

\section{Nature Based Algorithms}

The performance of the digital image watermarking can be improved to a great extent if we use the new approach in optimization techniques i.e. Nature based Algorithms. These algorithms are capable to optimize the complex problems of the watermarking. This section provides the overview of the current algorithms based on nature. 


\section{International Journal of Science and Research (IJSR) \\ ISSN (Online): 2319-7064}

Index Copernicus Value (2013): 6.14 | Impact Factor (2014): 5.611

\section{1) Firefly Algorithm}

Firefly algorithm is based on the behaviour of the fireflies. Yang in 2008 developed this algorithm at Cambridge University. FA is a new metaheuristic algorithm for optimization problem. The flashing behaviour of the fireflies inspired this algorithm. Here the main two terms used are Brightness and Attractiveness. These two are directly proportional to each other. That is brighter firefly will attract more fireflies towards itself hence its attractiveness is also greater. There are three basic rules of this algorithm which it follows:

A) All the fireflies are unisex, which means all the fireflies can attract every other firefly in the set of fireflies.

B) The attractiveness is directly proportional to the brightness and inversely proportional to the distance between fireflies. Hence a less bright firefly will move towards a brighter firefly. And if there is no such direction in which the brightness of the firefly increases then it will move randomly.

C) The brightness of the firefly is calculated on the basis of an objective function. Also the brightness is proportional to objective function.

The attractiveness, $\beta$ of a firefly can be calculated as:

$$
\mathrm{B}=\beta_{0} \mathrm{e}^{-\lambda \mathrm{r}}{ }_{\mathrm{ij}}
$$

Where $\beta_{0}$ is the attractiveness at $r_{i j}=0, \lambda$ is the light absorption coefficient and $r_{i j}$ is the distance between the fireflies $\mathrm{i}$ and $\mathrm{j}$.

The movement of a firefly towards a brighter firefly can be determined as:

$$
\mathrm{X}_{\mathrm{i}+1}:=\mathrm{x}_{\mathrm{i}}+\beta_{0} \mathrm{e}^{-\lambda \mathrm{r}}{ }_{\mathrm{ij}}^{2}\left(\mathrm{x}_{\mathrm{j}}-\mathrm{X}_{\mathrm{i}}\right)+\alpha \varepsilon(2)
$$

Where $\varepsilon$ is random numbers vector and $\alpha$ is a randomised parameter.

The working of the Firefly Algorithm based on the above rules is shown in the figure 1 .

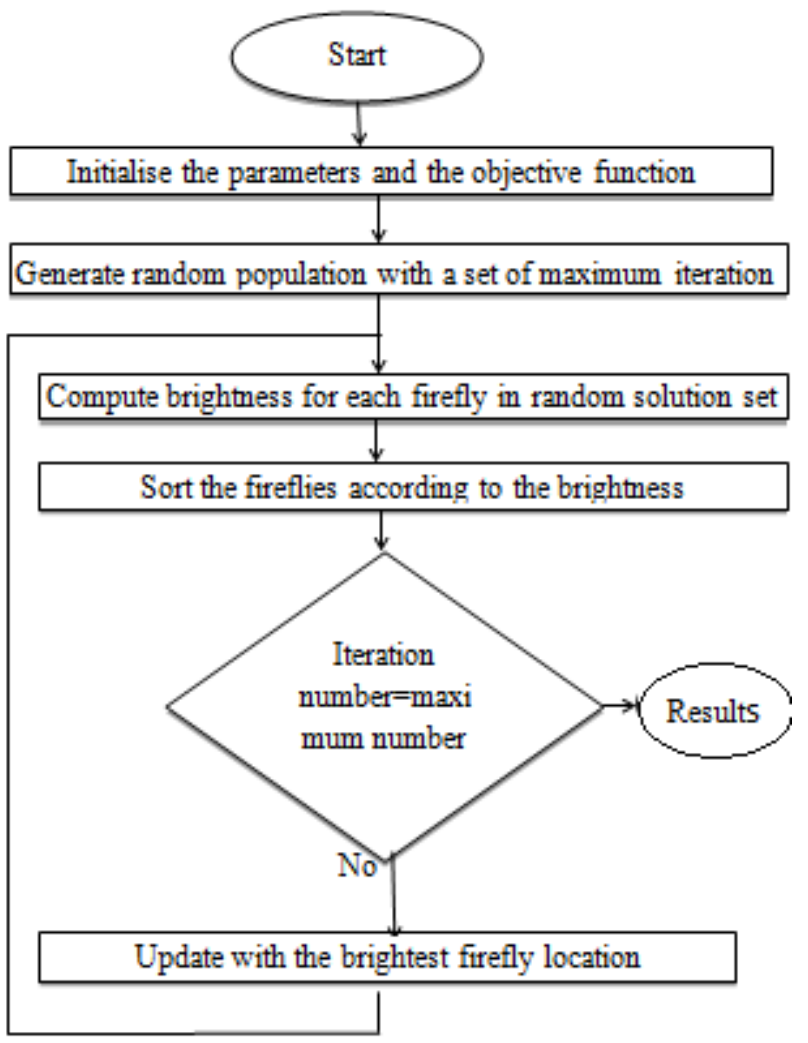

Figure 1

\section{2) Modified Firefly Algorithm}

The current global best solution can be obtained by the brightest firefly according to FA. However, if this brightest firefly moves in random direction as per the standard FA then, its brightness may decrease in a particular direction and hence, the performance of the algorithm degrades in that iteration. Now to reduce this degradation Modified Firefly Algorithm slightly changes this random movement. The new rule of movement is as follows:

The firefly will move only in a direction in which its brightness increases and if there is no such direction, then it will remain at its own position.

This slight change in the standard FA does not let the brightness decreases, hence the performance of the algorithm remain global best. The movement is done in a direction of brightest firefly by determining the randomly generated $\mathrm{m}$ unit vectors, $\mathrm{u}_{1}, \mathrm{u}_{2} \ldots . . \mathrm{u}_{\mathrm{m}}$. Now select the direction $u$ such that the brightness of the firefly increases in that particular direction. The movement of the brightness of the firefly can be determined as:

$$
\mathrm{x}:=\mathrm{x}+\alpha \mathrm{u}
$$

Where $\alpha$ is the random step length. If there is no such direction in which the brightness of the brightest firefly increases, then the firefly will remain at its current position.

\section{3) Cuttlefish Algorithm}

Cuttlefish Algorithm (CFA) is a new meta-heuristic optimization algorithm which is inspired by the changing of colour of the cuttlefish. The cuttlefish produces these colours and patterns through the reflection from its three layers of skin. CFA has two processes - Reflection and Visibility. Reflection is a process of simulating the reflection of light mechanism and visibility simulates the matching pattern "s visibility of the cuttlefish. The global optimal solution can be found using these two processes. The new best solution is determined by:

$$
\mathrm{n}_{\mathrm{p}}=\text { reflection }+ \text { visibility }
$$

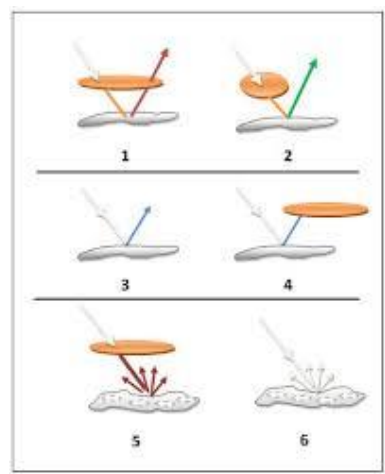

Figure 2: six cases

The CFA is implemented as shown in Figure 3. 


\section{International Journal of Science and Research (IJSR) \\ ISSN (Online): 2319-7064}

Index Copernicus Value (2013): 6.14 | Impact Factor (2014): 5.611

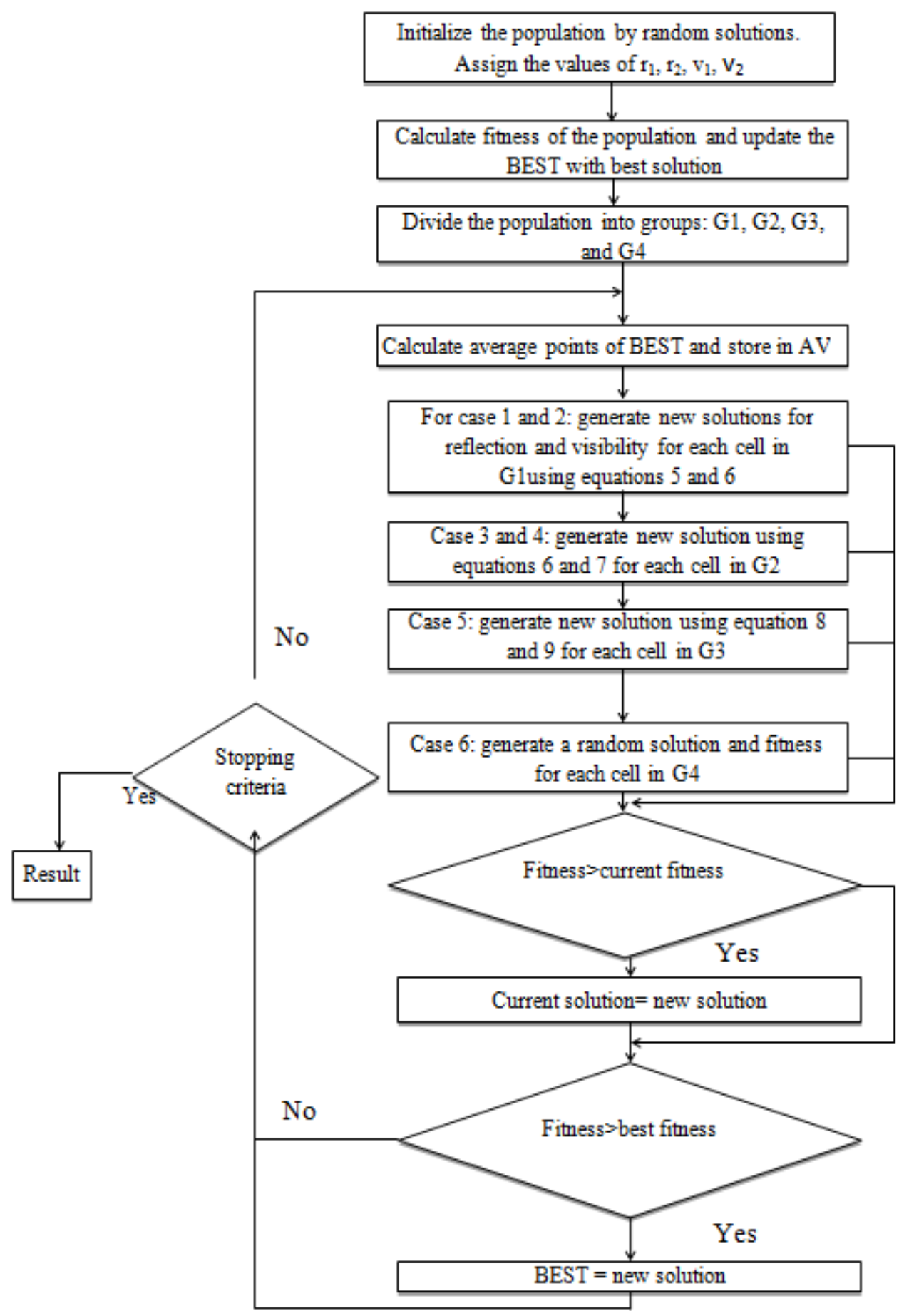

Figure 3

The CFA uses the basic working of the three layers of the skin cell of the cuttlefish, namely Chromatophores, leucophore and iridophore. It has two mechanism of contraction and shrinking of the skin. When the skin contract then it is stretched and the inside pigment covers a large surface and when these muscles relax then the saccule will shrink and hides the inside pigment. The algorithm is implemented by reordering of three layers of skin cells into six possible cases as shown in figure 2 .

Hence, for 6 cases, we have equations for reflection and visibility as:

$$
\text { Reflection }_{\mathrm{k}}=\mathrm{R} * \mathrm{G}_{1}[\mathrm{i}] \text {.points }[\mathrm{k}]
$$

Visibility $_{\mathrm{k}}=\mathrm{V}^{*}$ (best.points $[\mathrm{k}]-\mathrm{G}_{1}[\mathrm{i}]$.points $\left.[\mathrm{k}]\right)$
Where $G_{1}$ is the group of cell, $i$ is the $i^{\text {th }}$ cell of $G_{1}$, point $[k]$ is the $\mathrm{k}^{\text {th }}$ point of cell $\mathrm{I}$ and best solution is given by best.points. $\mathrm{R}$ is the parameter which finds the stretch and shrink interval and $\mathrm{V}$ is the visibility degree.

$$
\begin{aligned}
& \mathrm{R}=\text { Random }()^{*}\left(\mathrm{r}_{1}-\mathrm{r}_{2}\right)+\mathrm{r}_{2} \\
& \mathrm{~V}=\text { Random }()^{*}\left(\mathrm{v}_{1}-\mathrm{v}_{2}\right)+\mathrm{v}_{2}
\end{aligned}
$$

$\mathrm{r}_{1}, \mathrm{r}_{2}, \mathrm{v}_{1}, \mathrm{v}_{2}$ are specified by the user.

For case 5 we will use:

$$
\text { Reflection }_{\mathrm{k}}=\mathrm{R}^{*} \text { best.points }[\mathrm{k}]
$$

\section{4) Bat Algorithm}

(5) Bat algorithm (BA) is inspired by the Echolocation

(6) behaviour of bats. The bats produce sonar, called echolocation which is of very high pitch and frequency that 


\section{International Journal of Science and Research (IJSR) \\ ISSN (Online): 2319-7064}

Index Copernicus Value (2013): 6.14 | Impact Factor (2014): 5.611

cannot be heard by the human ears. They catch their prey using these sound waves. Bats produce sound waves and listen to them when they echo back after the reflection from a surface in the environment or the prey. They use frequency modulated signals and their bandwidth varies with the species. Usually the frequency range for all the bats is in between $25 \mathrm{kHz}$ to $150 \mathrm{kHz}$, but some species can emit higher than $150 \mathrm{kHz}$. Bats tend to emit about 10 to 20 sound waves every second in normal conditions but when they are in hunt of the prey this emission will go up to 200 per second. Same is the case with loudness, i.e. loudness is maximum when they are near the prey and minimum when they are just horning for the prey. Bats have an amazing capability to use the time delays between its ears and can estimate about the size of the prey, distance and even the moving speed of that prey. They use the phenomenon of Doppler Effect induced by the prey insects by fluttering of their wings. The BA is implemented as in Figure 4:

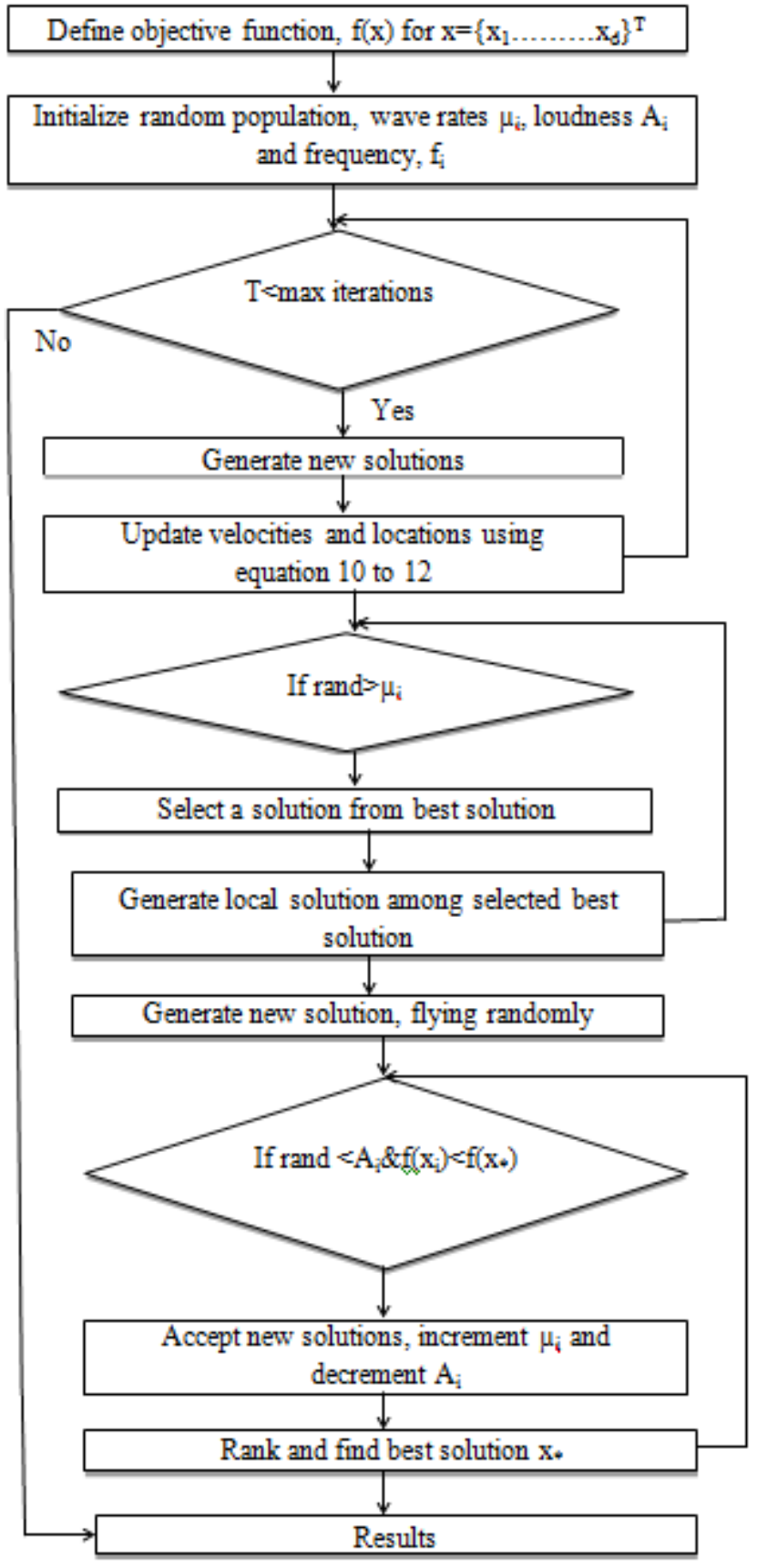

There are basically three rules to follow in BA these are:

a) Every bat uses the concept of echolocation and can sense the difference between a prey and normal environmental surrounding

b) All bats fly with a velocity $v_{i}$ at position $x_{i}$ randomly. It has a constant frequency say, $f_{\min }$ and a varying wavelength, $\lambda$ with loudness as $A_{0}$ while searching a prey. Depending on the contiguity, bats can change the wavelength or frequency of their emitted sound waves and adapt the rate of emission of these waves, $\mu \in[0,1]$, which is dependent on the closeness of the target

c) Assume that the loudness of the sound waves vary between a maximum of $A_{0}$ to minimum of $A_{\text {min }}$.

$$
\begin{gathered}
\mathrm{f}_{\mathrm{i}}=\mathrm{f}_{\min }+\left(\mathrm{f}_{\max }-\mathrm{f}_{\min }\right) \beta \\
\mathrm{v}_{\mathrm{i}}^{\mathrm{t}}=\mathrm{v}_{\mathrm{i}}^{\mathrm{t}-1}+\left(\mathrm{x}_{\mathrm{i}}^{\mathrm{t}-\mathrm{X} *}\right) \mathrm{f}_{\mathrm{i}} \\
\mathrm{x}_{\mathrm{i}}^{\mathrm{t}}=\mathrm{x}_{\mathrm{i}}^{\mathrm{t}-1}+\mathrm{v}_{\mathrm{i}}^{\mathrm{t}}
\end{gathered}
$$

where $x_{i}$ is the position and $v_{i}$ is the velocity in $d$ dimensional space. $\mathrm{x}_{\mathrm{i}}^{\mathrm{t}}$ and $\mathrm{v}_{\mathrm{i}}^{\mathrm{t}}$ are position and velocity at time $t$ respectively. A random vector, $\beta €[0,1]$ is drawn from a uniform distribution. $\mathrm{X}_{*}$ is the current best solution

new solution for each bat,

$$
\mathrm{X}_{\text {new }}=\mathrm{X}_{\text {old }}+\varepsilon \mathrm{A}^{\mathrm{t}}
$$

where $\varepsilon$ is a random number from $[-1,1]$ and $A^{t}$ is the average loudness.

\section{5) Artificial Bee Colony}

Artificial bee colony algorithm (ABC) is inspired by the combing behaviour of the honey bees. It has been developed by the Karaboga as an optimization algorithm for numerical problems. $\mathrm{ABC}$ can be used in the problem of optimization in digital image watermarking. According to ABC algorithm the honey bees are divided into three groups: Inked, bystanders and recruiters. Half of the bees are inked and half are bystanders. There is an equal food source around the hive as inked bees. The recruiters are those bees who abandon their food sources. The food sources here represent the possible solution and the amount of food source represents the fitness or quality of the solutions. The number of the inked bees or bystander bees always depend on the number of solutions, thus they are always equal the number of solutions. The probability can be calculated for a food source in order to be selected by the bystander bees:

$$
p_{i}=\frac{f i t_{i}}{\sum_{n=1}^{S N} f i t_{n}}
$$

Where fit $t_{i}$ is the fitness of the generated solution and SN is the amount of food source.

To calculate the candidate food source position from te previous value:

$$
\mathrm{V}_{\mathrm{i}, \mathrm{j}}=\mathrm{x}_{\mathrm{i}, \mathrm{j}}+\Phi_{\mathrm{i}, \mathrm{j}}\left(\mathrm{x}_{\mathrm{i}, \mathrm{j}} \mathrm{x}_{\mathrm{k}, \mathrm{j}}\right)
$$

Where $\mathrm{k} \in\{1$......SN $\}$ and $\mathrm{j} \in\{1,2 \ldots \ldots \ldots . \mathrm{D}\}$ are indexes chosen randomly. $\Phi_{\mathrm{i}, \mathrm{j}}$ is a random number and lies between 1 and 1.

To calculate new position for the abandoned food source and then replacing with older value we have:

$$
X_{i}^{j}=X_{\text {min }}{ }^{j}+\operatorname{rand}(0,1)\left(x_{\max }{ }^{j}-X_{\text {min }}{ }^{j}\right)
$$

Figure 4: 


\section{International Journal of Science and Research (IJSR) ISSN (Online): 2319-7064}

Index Copernicus Value (2013): 6.14 | Impact Factor (2014): 5.611

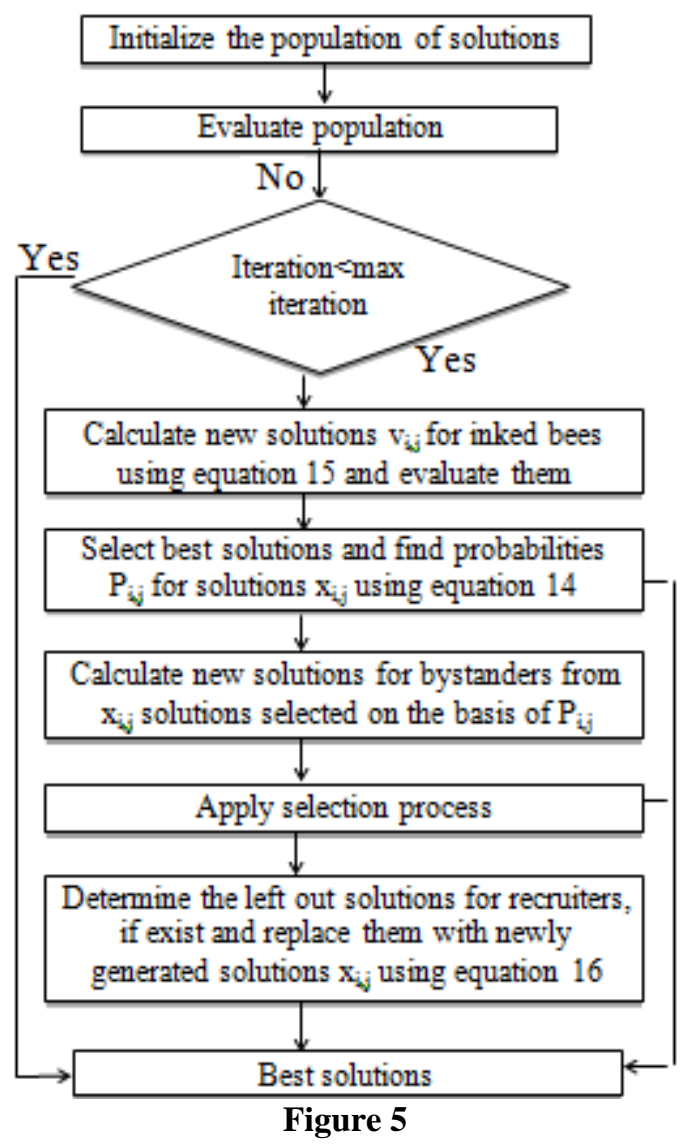

\section{6) Seed Based Plant Propagation Algorithm}

The plant propagation algorithm (PPA) is mainly derived from the Strawberry plants, hence also known as Strawberry Algorithm. It has inherited its process by the property of plants to propagate using Runners. The important advantage of this algorithm is that it can be implemented for any optimization problem. It will give the desired solution to all these problems. Seed based plant propagation algorithm (SBPPA) uses the basic concept of PPA with a slight change in the propagation. Here, the propagation is done by the seeds. A feeding station is built to attract the attention of the agents who helps in dispersing the seeds. This feeding station is important to build as the fruits and seeds are produced periodically by the plants. Many plants and depends on each other to preserve their species. Hence, some plants require their seeds to be dispersed well by the dispersing agents like frugivorous animals and birds. This dispersal of seeds by these agents is also referred to as Seed Shadow. The feeding model has two parts:

a) The rate of visiting a plant by the agents is dependent on the quantity of the fruit or seed on that plant.

b) A probability density function, which implies the rate at which the agents are being served by the plants with the fruits or seeds.

This model calculates the quantity of seeds dispersed locally and globally. There are two basic concepts which needs to be balanced for effective implementation of SBPPA: exploitation or intensification which searches locally and converge optimally to local solution and exploration or diversification allows for global search and finds the global optimum solution. This algorithm assumes that the arrival of the agents to feed themselves on the plants is according to

the Poisson"s distribution. Let $\mathrm{X}^{\text {ec }}$ be denoting number of agents arriving per unit of time, $t$. Now $k$ arrivals in time $t$ can be formulated as :

$$
\mathrm{P}\left(\mathrm{X}^{\mathrm{ce}}=\mathrm{k}\right)=\left((\lambda \mathrm{t})^{\mathrm{k}} \mathrm{e}^{-\lambda t}\right) / \mathrm{k} \text { ! }
$$

Where, $\lambda$ is the mean rate of arrival of the agents per time unit

The SBPPA can be used in watermarking to find the optimal positions for the embedding of the watermark mage in the host image by finding the dispersion of pixels in the host image.

The SBPPA is described in Figure 7:

Here NP is population size, $r$ is the no. of iteration and max is the max iteration.

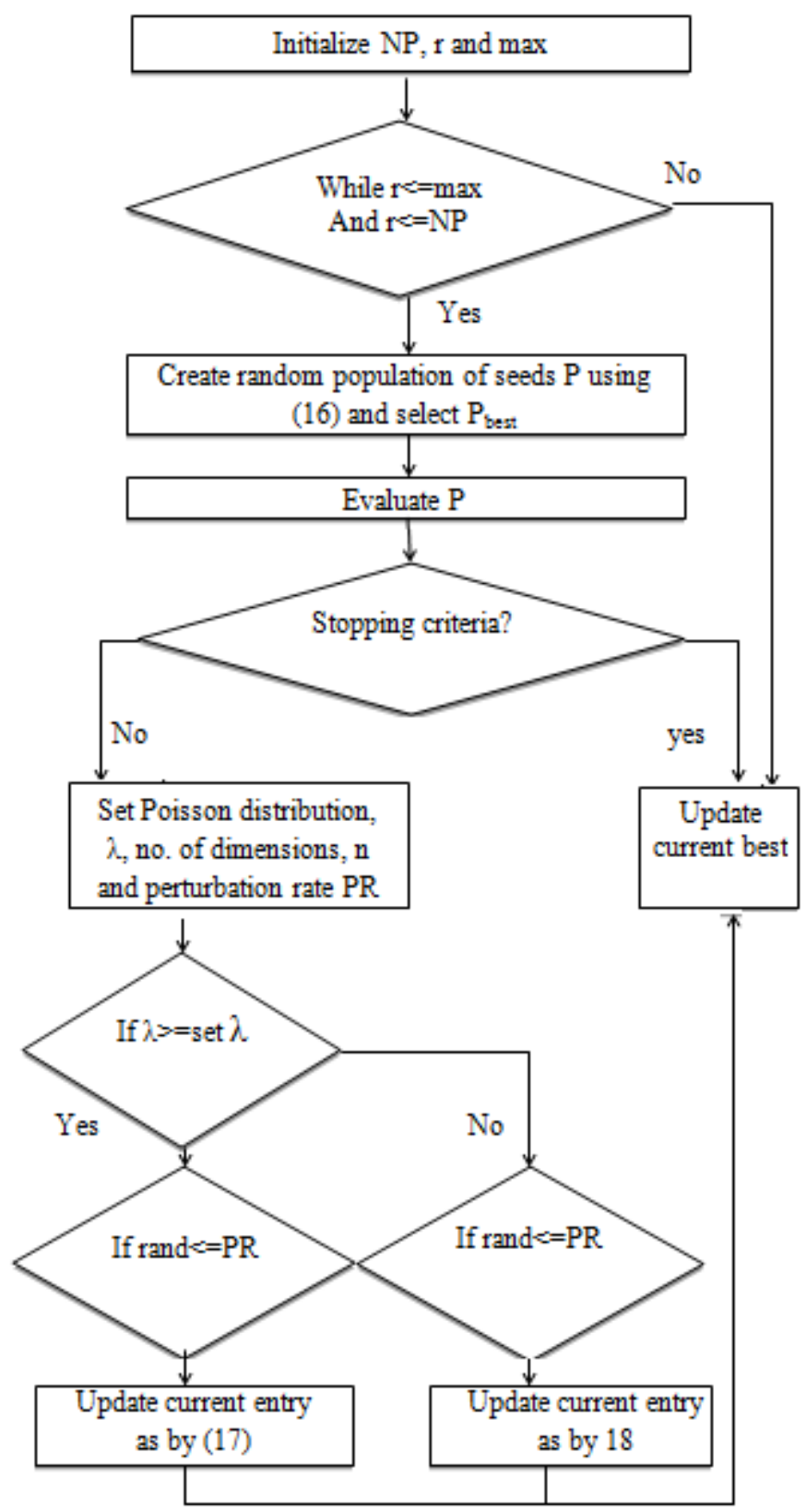

Figure 7

In case when Poisson distribution, $\lambda$ is less than the set value then the exploitation will occur and the algorithm will search locally: 


\section{International Journal of Science and Research (IJSR) \\ ISSN (Online): 2319-7064}

Index Copernicus Value (2013): 6.14 | Impact Factor (2014): 5.611

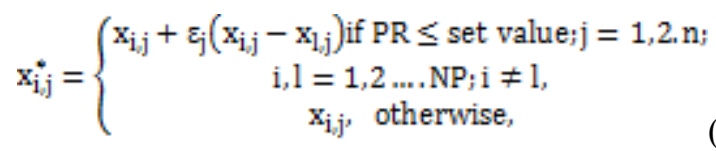

Where, $x_{i, j}^{*}$ and $x_{i, j} \in\left[a_{j} b_{j}\right]$ are the coordinates of the seeds $\mathrm{X}_{\mathrm{i}}{ }^{*}$ and $\mathrm{Xi}$ respectively at $\mathrm{j}^{\text {th }}$ position, $\mathrm{a}_{\mathrm{j}}$ and $\mathrm{b}_{\mathrm{j}}$ are the lower and upper bound of $\mathrm{j}^{\text {th }}$ coordinate.

And when $\lambda$ is greater than a defined value then the exploration will occur and the algorithm will search globally:

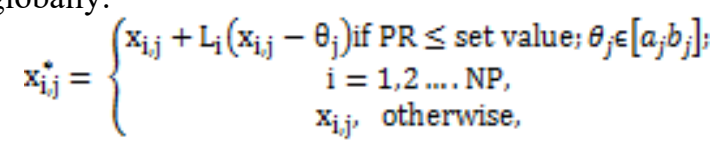

The random population of NP seeds is generated initially using:

Where, $x_{i, j} \in\left[a_{j} b_{j}\right]$ and $\eta_{j} \in(0,1)$.

$$
x_{i, j}=a_{j}+\left(b_{j}-a_{j}\right) \eta_{j}, j=1,2 \ldots \ldots n
$$

\section{Conclusion}

There are many algorithms which can be used in the optimization of the digital image watermarking and each has its own advantages and disadvantages. No particular algorithm is capable of resisting all kind of attacks; one algorithm may be resistant to some attacks but may not provide robustness to some other attacks. In the previous sections algorithms based on nature has been presented with their flow of steps and defining the calculations used in the implementation of these algorithms. However, these algorithms are not exposed much to the watermarking industry and researchers; hence it is of great interest to use these algorithms in the future. It is expected from the future researchers to enhance the use of nature based algorithms in the field of digital image watermarking optimization and newer techniques would also be implemented.

\section{References}

[1] Hartung, F., and Kutter, M. 1999. Multimedia Watermarking Techniques, Proceeding of IEEE, 87(7) 1079-1107, July 1999.

[2] Liu, F., \& Liu, Y. (2008). "A watermarking algorithm for digital image based on DCT and SVD." In IEEE Congress on image and signal Processing (Vol. 1, pp. 380-383). Sanya, Hainan, China.

[3] Lai, C.-C. (2011). "A digital watermarking scheme based on singular value decomposition and tiny genetic algorithm.” Digital signal processing. Elsevier. 522-527.

[4] Ghazy, R., El- Fishawy, N., Hadhoud, M., Dessouky, M.m \& El-Samie, F. (2007). "An efficient block by block SVD based image watermarking scheme." In proceedings of the 24th national radio science conference (pp. 1-9), Cairo, Egypt

[5] K. Vijaya Durga, G.Mamatha, Ch.Hima Bindu (2015) "SVD Based Image Watermarking With Firefly Algorithm". In International Conference on Computer Communication and Informatics, Coimbatore, INDIA

[6] Veysel Aslantas (2009). "An optimal robust image watermarking based on SVD using differential evolution algorithm". In Optics communications Elsevier 769-777.
[7] Jain, C., Arora, S., \&Panigrahi, P.K., (2008). "A reliable SVD based watermarking scheme," adsabs.harvad.edu/abs/2008arXiv 0808.0309J.

[8] Chandra, D. (2002). "Digital image watermarking using singular value decomposition." In proceedings of the IEEE 45th Midwest symposium on circuits and systems (Vol.3, pp. 264-267). USA: Oklahoma State University.

[9] Surafel Luleseged Tilahun and Hong Choon Ong.,(2012). "Modified Firefly Algorithm”. In Hindawi publishing corporation, journal of applied mathematics volume 2012, article 467631.

[10]P. Surekha and Sumathi (2011). "Implementation of genetic algorithm for a dwt based image watermarking scheme". ICTACT journal on soft computing:special issue on fuzzy in industry and process automation, volume 02 , issue 01 .

[11]S. Lee, D. Jang, C.D. Yoo, AN SVD-based watermarking method for image content authentication with improved security, in: Proc. ICASSP05, 2005, pp. 525-528.

[12] Yuan, Q.; C. Li; and Y. Zhong. 2007. Adaptive DWTSVD domain image watermarking using a human visual model, Proceedings of the 9th Advanced Communication Technology Conference 3: 1947-1951.

[13] B. Kim, J. G. Choi and D. Min, "Robust Digital Watermarking Method Against Geometric Attacks," Real Time Imaging Processing, Vol. 9, No. 2, 2003, pp. 139149.

[14]E. Vahedi, R. A. Zoroofi and M. Shiva, "Toward a new wavelet-based watermarking approach for color images using bio-inspired optimization principles", Digital Signal Processing, vol. 22, no. 1, (2012), pp. 153-162.

[15]X.-S. Yang, "Engineering optimization via natureinspired virtual bee algorithms", Artificial Intelligence and Knowledge Engineering Applications: A Bioinspired Approach, Lecture Notes in Computer Science, vol. 3562, (2005), pp. 317-323.

[16][50] D. Karaboga, "An idea based on honey bee swarm for numerical optimization", Technical Report TR06, Erciyes University, Turkey, (2005).

[17] Y. Chen, W. Yu and J. Feng, "A Reliable SVD-DWT Based Watermarking Scheme with Artificial Bee Colony Algorithm", International Journal of Digital Content Technology and its Applications, vol. 6, no. 22, (2012), pp. 430-439.

[18]X.-S. Yang, "Nature-Inspired Metaheuristic Algorithms", Luniver Press, UK, (2008).

[19]X.-S. Yang and X. He, „Firefly Algorithm: Recent Advances and Applications", Int. J. Swarm Intelligence, vol. 1, no. 1, (2013), pp. 36-50.

[20] C. Agarwal, A. Mishra, A. Sharma and G. Chetty, "A Novel Image Watermarking Scheme using firefly Algorithm", International Conference on Artificial Intelligence and software Engineering, (2014), pp. 430436.

[21] A. Salhi and E. S. Fraga, "Nature-inspired optimisation approaches and the new plant propagation algorithm," in Proceedings of the International Conference on Numerical Analysis and Optimization (ICeMATH " 11 ), pp. K2-1-K2-8, Yogyakarta, Indonesia, 2011.

[22] M. Sulaiman, A. Salhi, B. I. Selamoglu, and O. B. Kirikchi, "A plant propagation algorithm for constrained engineering optimisation 


\section{International Journal of Science and Research (IJSR) \\ ISSN (Online): 2319-7064}

Index Copernicus Value (2013): 6.14 | Impact Factor (2014): 5.611

problems,"Mathematical Problems in Engineering, vol. 2014, Article ID627416, 10 pages, 2014.

[23] Abdel-Rahman, E. M., Ahmad, A. R., Akhtar, S., (2012). A metaheurisic bat- inspired algorithm for full body human pose estimation, in: Ninth Conference on Computer and Robot Vision, pp. 369-375.

[24] Bora, T. C., Coelho, L. S., Lebensztajn, L., (2012). Batinspired optimization approach for the brushless DC wheel motor problem, IEEE Trans. Magnetics, Vol. 48, No. 2, 947-950 (2012).

[25] Adel Sabry Eesa, Adnan Mohsin Abdulazeez, Zeynep Orman, Cuttlefish Algorithm - A Novel Bio-Inspired Optimization Algorithm, International Journal of Scientific and Engineering Research, Vol. 4, Issue 9,September, 2013.

[26] R. A. Cloney, and S. L. Brocco, Chromatophore organs, reflector cells, iridocytes and leucophores in cephalopods, Am. Zool. 1983. 